\title{
Thermal decohesion model validity for polycrystalline advanced ceramics
}

Marin Petrovic*, Elvedin Kljuno

Mechanical Engineering Faculty, University of Sarajevo, Sarajevo, Bosnia and Herzegovina

\section{ARTICLE INFO}

\section{Article history:}

Received 23 March 2017

Received in revised form

8 May 2017

Accepted 18 May 2017

\section{Keywords:}

Experimental mechanics

Advanced ceramics

Brittle fracture

Fracture mechanics

Thermal decohesion model

\begin{abstract}
A B S T R A C T
Polycrystalline advanced ceramics is a synthetic product produced by sintering together selected carbide or other tough material grains in a metal matrix. Due to wide and sensitive application of these materials, the accurate and efficient determination of the associated fracture mechanisms is of fundamental importance to material manufacturers and end users alike. An experimental investigation of two different grades of advanced ceramics was performed. The material was found to follow a thermal-decohesion model suggesting that adiabatic conditions occur at the crack tip during fracture.
\end{abstract}

(C) 2017 The Authors. Published by IASE. This is an open access article under the CC BY-NC-ND license (http://creativecommons.org/licenses/by-nc-nd/4.0/).

\section{Introduction}

Polycrystalline advanced ceramics is a synthetic product produced by sintering together selected carbide or other tough material grains in a metal matrix. Random orientation of the grains enables extremely high hardness and abrasion resistance in all directions. As these materials are valued for their abrasion resistance, they find use in abrasive environments such as the wear plates of crushing equipment in mining operations where other ceramic materials would not be suitable. Advanced ceramics are also used in the medicine, electrical and aerospace industries.

Due to wide and sensitive application of these materials, the accurate and efficient determination of the associated fracture mechanisms is of fundamental importance to material manufacturers and end users alike. The ability to predict the behaviour of such materials can lead to better designs, increased performance, reduced costs and enhanced safety.

Published fracture data for polycrystalline advanced ceramics is relatively scarce and somewhat limited in nature. On one hand, the current situation offers great opportunities for testing and improvement of these materials, but on the other hand, it is quite difficult to measure the properties of these very hard and brittle materials at elevated temperatures. In addition, the costs involved in research of this kind are very high, which

\footnotetext{
* Corresponding Author.

Email Address: petrovic@mef.unsa.ba (M. Petrovic) https://doi.org/10.21833/ijaas.2017.07.001

2313-626X/C) 2017 The Authors. Published by IASE. This is an open access article under the CC BY-NC-ND license (http://creativecommons.org/licenses/by-nc-nd/4.0/)
}

is main reason why a systematic study of this kind has not been performed to date.

The findings presented in this paper lead to a greater understanding of the fracture behaviour of polycrystalline advanced ceramics, informing the development of improved future grades of materials.

\section{Materials and methods}

\subsection{Materials and specimens}

Two grades of polycrystalline advanced ceramics have been used in this analysis containing different mean grain sizes and amounts of metal matrix phase employed as a binder in the structure. These grades of material will be referred to as grade $A$ and grade $B$ and contain average grains of 6 and $30 \mu \mathrm{m}$, respectively.

As the grade A material has more grain boundaries, a higher percentage of cobalt binder is expected.

Single edge $V$ notched beam (SEVNB) specimens were cut from bulk circular disks with a diameter of $50 \mathrm{~mm}$ using a laser. The test specimens had the following average dimensions:

- 'thick' samples: (b) $4.73 \mathrm{~mm} \times(h) 6.22 \mathrm{~mm} \times(I)$ $28.5 \mathrm{~mm}$

- 'thin' samples: (b) $2.98 \mathrm{~mm} \times(h) 6.22 \mathrm{~mm} \times(l) 28.5$ $\mathrm{mm}$.

The need for small-sized specimens arises for several reasons, but mainly due to the high cost of the material. A schematic of the test specimen is given in Fig. 1. The depths of the notches were nominally (a) $1.25 \mathrm{~mm}$ with the notch angle $45^{\circ}$. 


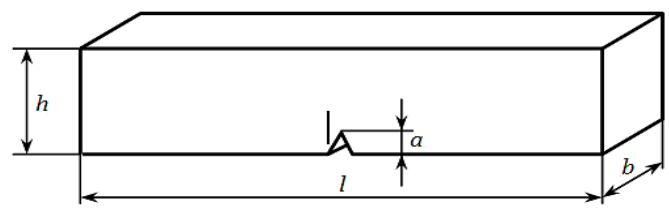

Fig. 1: Sample geometry

\subsection{Testing environment}

The specimens were positioned on a three-point bend (TPB) fixture with a span $(S)$ of $24.8 \mathrm{~mm}$ and two supporting rollers with a radius of $3 \mathrm{~mm}$. The load was applied by a stainless steel striker fitted with a loading roller with the same radius of $3 \mathrm{~mm}$. All rollers were made from Inconel alloy 600 (Ni72/Cr16/Fe8) which retains its stiffness at elevated temperatures. Tests were carried out at a range of loading rates: $1 \mathrm{~mm} / \mathrm{min}$ and $100 \mathrm{~mm} / \mathrm{min}$ on a standard screw driven testing machine, and 0.3 $\mathrm{m} / \mathrm{s}, 1 \mathrm{~m} / \mathrm{s}$ and $5 \mathrm{~m} / \mathrm{s}$ on an instrumented dropweight tower. Three temperature levels were employed: 25,300 and $600^{\circ} \mathrm{C}$.

In order to perform tests at elevated temperatures, a high temperature testing chamber was constructed (Petrovic et al., 2012). A stainless steel block with dimensions of $450 \mathrm{~mm} \times 450 \mathrm{~mm} \times$ $25 \mathrm{~mm}$ was cut and a hole of $12 \mathrm{~mm}$ in depth with the diameter of $40 \mathrm{~mm}$ was drilled in the centre to serve as a support for the three-point bend rig. The insulation consisted of ceramic fibre boards, exhibiting high temperature stability for continuous use at temperatures up to $1430^{\circ} \mathrm{C}$. Temperature in this chamber can be regulated with the precision of $\pm 2^{\circ} \mathrm{C}$. During loading, the striker attached to the testing machine was entering the furnace through a small rectangular opening of $10 \mathrm{~mm} \times 30 \mathrm{~mm}$ on the lid. A CAD representation of the furnace interior is given in Fig. 2.

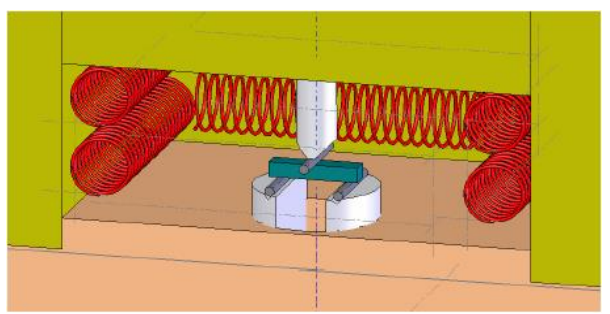

Fig. 2: CAD representation of the furnace interior with TPB sample in place

\subsection{Low-rate tests}

The low-rate three point bend flexural tests were performed using a standard screw-driven tensile testing machine (Petrovic et al., 2009; Petrovic et al., 2011). The plane strain fracture toughness is determined by the load at initiation method according to ASTM E1820-01 fracture standard:

$K_{I c}=\left(P_{i n} S / b h^{1.5}\right) f(\alpha)$

where $\alpha=a / h, P_{\text {in }}$ is the breaking load and $f(\alpha)$ is a fitting function of $\alpha$ as outlined in (Petrovic et al., 2011).
The load versus crosshead displacement at the loading point is recorded automatically by the testing machine in low rate tests. The fracture initiation corresponds to the peak load point after which the load drops suddenly. For improved accuracy, for tests at $100 \mathrm{~mm} / \mathrm{min}$ and above, specimens were instrumented with strain gauges bonded as close to the crack tip as possible, in order to determine the initiation load, and therefore fracture toughness, directly from the strain values measured by the gauge.

\subsection{High-rate tests}

High rate tests were carried out on a drop-weight tower. In these tests the point of initiation cannot be accurately detected from the load signals measured by the machine load cell at high rates. Various dynamic effects occur in the impact test and therefore it is very difficult to determine the initiation time from the load-time curve obtained by the machine load cell, as peaks in this curve are due to the dynamics of the system and are not necessarily related to the crack initiation.

Also, the measured striker load will be different to the crack tip load. It was shown (Kalthoff, 1985) that the dynamic effects at the crack tip were significantly smaller than the load measured at the striker contact point. This means that expensive instrumentation of the specimen was necessary and the load-time trace from the crack-tip strain gauge must be used for high rate tests (Carolan et al., 2010).

\section{Results and discussion}

\subsection{Apparent fracture toughness}

A total of 108 specimens were tested in the threepoint bending configuration at quasistatic and dynamic rates and three temperature levels, $25^{\circ} \mathrm{C}$, $300^{\circ} \mathrm{C}$ and $600^{\circ} \mathrm{C}$, and the fracture toughness was evaluated according to the static formula. Experimental results from these tests on thick and thin specimens are summarised in Fig. 3 and Fig. 4, respectively. Unfortunately, it was unfeasible to obtain results across the full range of testing conditions for both types of specimens due to their limited supply. However, the number of available specimens and obtained results were quite sufficient to quantify the effects of different parameters on the fracture toughness and draw important conclusions.

Test results in Fig. 3 revealed the apparent fracture toughness of grade A material to remain generally constant at quasi-static rates, and then to decrease with a further increase in the loading rate above $100 \mathrm{~mm} / \mathrm{min}$. Fig. 4 shows that the apparent fracture toughness of the grade $B$ material remains relatively constant at quasi-static and intermediate rates up to $0.3 \mathrm{~m} / \mathrm{s}$, and then sharply drops at higher rates. 


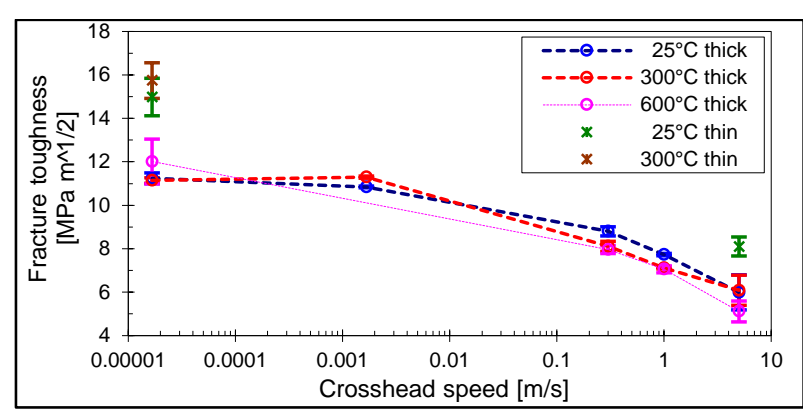

Fig. 3: Apparent fracture toughness of grade $A$ as function of crosshead speed and temperature

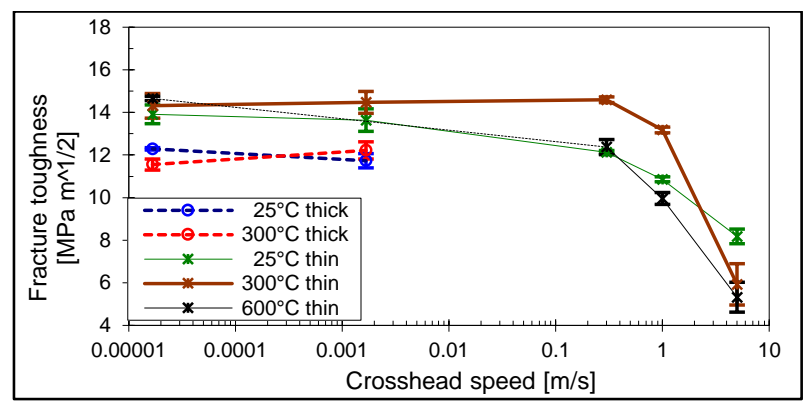

Fig. 4: Apparent fracture toughness of grade $B$ as function of crosshead speed and temperature

\subsection{Thermal decohesion model}

When the compliance of the test machine and loading rig were taken into account, the deflection of the specimen was an order of magnitude smaller than the crosshead displacement and so also were the actual loading rates. The local loading rate $v_{l}$ was calculated numerically using finite volume stress analysis. Here, the numerical strain output was compared to the experimental strain signal and the loading rate was varied until the appropriate agreement was obtained. Fracture initiation times, measured from the instant of contact to fracture initiation, for the two tested grades are shown as a function of actual local loading rates in Fig. 5. The initiation time values at dynamic loading rates decrease almost linearly in the double logarithmic plot, regardless of the testing temperature and the sample thickness, returning a slope of approximately $-4 / 3$ for each grade of material for local loading rates of $3 \times 10^{-4} \mathrm{~m} / \mathrm{s}$ and above. The $-4 / 3$ slope is a very good fit for grade $A$ and to a lesser extent for grade $B$ for nominal loading rates above $100 \mathrm{~mm} / \mathrm{min}$.

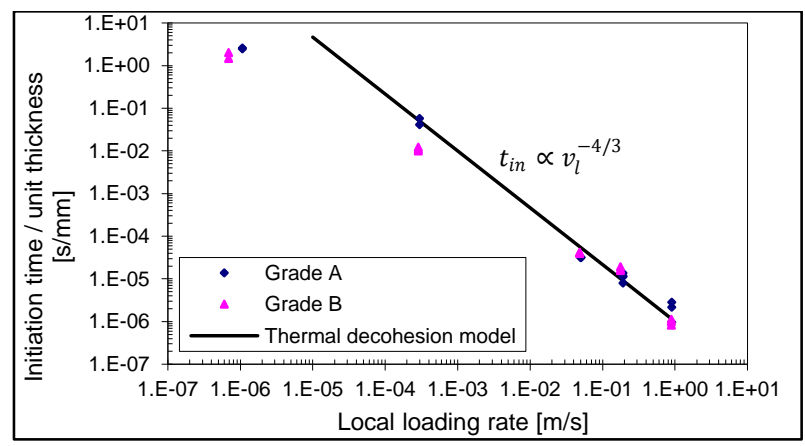

Fig. 5: Fracture initiation times vs. local loading rate for grade $\mathrm{A}$ and grade $\mathrm{B}$
This behaviour can be explained with reference to a thermal model (Williams and Hodgkinson, 1981; Rager, 2003), which indicates that the fracture process could be thermally initiated by heat trapped in a small zone around the crack tip at high impact velocities due to extremely small time scales for crack initiation and propagation. This means that adiabatic conditions take place at the crack tip during fracture, increasing the temperature in the vicinity of the crack tip. This temperature rise can be determined according to the Carslaw and Jaeger (1959) solution of the heat transfer equation, using a fixed rate of energy input $G_{c} / t=$ const (Williams and Hodgkinson, 1981) for the purely adiabatic case:

$\Delta T(t)=G_{c} /\left(\pi \rho_{C o} c_{p} k t_{i n}\right)^{1 / 2}$

where, $G_{c}$ is the critical fracture energy, $\rho_{\text {co }}$ is mass density of cobalt, $c_{\mathrm{p}}$ is specific heat capacity, $\mathrm{k}$ is the thermal conductivity and tin is the fracture initiation time. Upon insertion of appropriate values for polycrystalline advanced ceramics obtained from experimental tests at $5 \mathrm{~m} / \mathrm{s}$ and $25^{\circ} \mathrm{C}\left(G_{\mathrm{c}}=100 \mathrm{~N} / \mathrm{m}\right.$, $\left.t_{\text {in }}=3.75 \mu \mathrm{s}\right)$ and physical properties of cobalt $\left(\rho_{\text {co }}=\right.$ $\left.8800 \mathrm{~kg} / \mathrm{m}^{3}, c_{\mathrm{p}}=420 \mathrm{~J} / \mathrm{kgK}, \mathrm{k}=92 \mathrm{~W} / \mathrm{mK}\right)$, assuming cobalt to be mainly responsible for trapping the heat, a temperature rise of around $2{ }^{\circ} \mathrm{C}$ is calculated. Considering the very significant difference between the coefficients of linear thermal expansion of the material of the grains and the cobalt (roughly ten times), a small change in temperature causes a considerable change in the relative expansion of these two materials. Also, considering the extremely high Young's modulus of the polycrystalline advanced ceramics, the calculated change in temperature induces about $20 \mathrm{MPa}$ of stress locally in the ligament of the binder between the grains. The presence of these thermal stresses would then result in dynamic fracture taking place at a reduced external load, ensuring that dynamic fracture toughness $K_{\mathrm{Id}}$ is less than the quasistatic $K_{\mathrm{Ib}}$ value. Fracture initiation times at a nominal loading rate of $100 \mathrm{~mm} / \mathrm{min}$ for the grade A material still follow the $-4 / 3$ slope, while tin at this loading rate for grade $B$ as well as for both materials at 1 $\mathrm{mm} / \mathrm{min}$ deviate from this line, which is reasonable due to the relatively large time scales available for heat conduction indicating that adiabatic conditions no longer dominate at the crack tip.

It is worth noting that even higher thermal stresses would be obtained if the shorter "damage time" (time from start of damage to complete separation as can be estimated from the numerical fracture model) was employed in Eq. 2 instead of the initiation time.

Looking into both graphs in Fig. 3 and Fig. 4, two distinct regions can be observed. At lower loading rates isothermal conditions predominate due to the extremely high conductivity of the material and considering that the loading process is ongoing for a relatively long time period. On the other hand, at higher loading rates adiabatic conditions prevail due to short loading times and the material deteriorates 
at the crack tip causing a dramatic drop in fracture toughness. The isothermal region is longer for the grade $\mathrm{B}$ material due to its higher conductivity which comes from the larger grain size and hence smaller binder content.

\section{Conclusion}

Polycrystalline advanced ceramics is finding increased application in industry, where brittle fracture can often render the tool unfit for purpose. In practical applications, the replacement of the tool is a time-consuming and costly exercise. The characterisation of its fracture properties is therefore of fundamental importance for the classification of existing materials and the development of improved grades in the future. The present paper has presented an analysis of the experimental results of the fracture behaviour of polycrystalline advanced ceramics specimens subjected to a range of loading rates and a range of temperatures, leading to the determination of the relevant fracture mechanisms.

At quasistatic rates the fracture toughness was found to be relatively constant. There is enough time for the heat generated at the crack tip to be conducted into the bulk material, so the process is essentially isothermal. At dynamic rates the fracture toughness drops consistently, most likely due to a temperature increase at the crack tip. Because of the extremely small time scales available for fracture initiation and propagation, heat generated in the small region ahead of the crack tip becomes trapped and adiabatic conditions take place, which is suggested by the fact that material satisfies an adiabatic thermal decohesion model. This results in the temperature and temperature gradients in this region becoming high, leading to the deterioration and damage of the metal matrix phase due to differences in the coefficients of thermal expansion of the grains and the binder, and a corresponding decrease in fracture toughness.

Apart from the theory of adiabatic heating in the vicinity of the crack tip resulting in lower $K_{\text {Ic }}$ values at high loading rates, it is also known that cobalt, like most metals with a face centred cubic microstructure, is very rate sensitive with the flow stress seen to be an increasing function of strain rate (Karimpoor et al., 2003; Paul et al., 2009; Kapoor et al., 2009). Therefore, the faster it is loaded, the more brittle it behaves, and the rate dependency in polycrystalline advanced ceramics is mainly due to the influence of the cobalt binder phase. Considering all these findings, it may be concluded that the cobalt binder phase is responsible for the deterioration in fracture toughness of polycrystalline advanced ceramics sintered compacts at high loading rates.

Continued investigation will lead to future insights into the dominant failure mechanisms and allow the development of an accurate roadmap for future material design.

\section{List of Symbols}

$a \quad$ initial crack length [mm]

$b \quad$ breadth of specimen [mm]

$c_{\mathrm{p}} \quad$ specific heat capacity $[\mathrm{J} / \mathrm{kgK}]$

$G_{\text {c }} \quad$ critical fracture energy $[\mathrm{N} / \mathrm{m}]$

$h \quad$ height of specimen [mm]

$k \quad$ thermal conductivity [W/mK]

$K_{\mathrm{Ib}} \quad$ measured fracture toughness [MPa ${ }^{1 / 2}$ ]

$K_{\text {Id }} \quad$ dynamic fracture toughness [MPa $\left.{ }^{1 / 2}\right]$

$l \quad$ length of specimen [mm]

$P_{\text {in }} \quad$ fracture initiation load [N]

$S \quad$ span of three-point bend test [mm]

$t_{\text {in }} \quad$ fracture initiation time $[\mu s]$

$v_{l} \quad$ local loading rate $[\mathrm{m} / \mathrm{s}]$

$\alpha \quad$ initial crack length to specimen height ratio

$\rho_{C o}$ mass density of cobalt $\left[\mathrm{kg} / \mathrm{m}^{3}\right]$

\section{References}

Carolan D, Petrovic M, Ivankovic A, and Murphy N (2010). Fracture properties of PCBN as a function of loading rate. Key Engineering Materials, 417(418): 669-672.

Carslaw HS and Jaeger JC (1959). Conduction of heat in solids. Oxford Clarendon Press, Oxford, UK.

Kalthoff JF (1985). On the measurement of dynamic fracture toughness-A review of recent work. International Journal of Fracture, 27(3): 277-298.

Kapoor R, Paul B, Raveendra S, Samajdar I, and Chakravartty JK (2009). Aspects of dynamic recrystallization in cobalt at high temperatures. Metallurgical and Materials Transactions A, 40(4): 818-827.

Karimpoor AA, Erb U, Aust KT, and Palumbo G (2003). High strength nanocrystalline cobalt with high tensile ductility. Scripta Materialia, 49(7): 651-656.

Paul B, Kapoor R, Chakravartty JK, Bidaye AC, Sharma IG, and Suri AK (2009). Hot working characteristics of cobalt in the temperature range 600-950 C. Scripta Materialia, 60(2): 104107.

Petrovic M, Carolan D, Ivankovic A, and Murphy N (2011). Role of rate and temperature on fracture and mechanical properties of PCD. Key Engineering Materials, 452(453): 153-156.

Petrovic M, Carolan D, Kanyanta V, and Ivankovic A (2009). Fracture and mechanical properties of PCBN as a function of loading rate and temperature. In the $6^{\text {th }}$ International Congress of Croatian Society of Mechanics (ICCSM'09). Dubrovnik, Croatia.

Petrovic M, Voloder A, and Ismic Dz (2012). Young's modulus of polycrystalline diamond as a function of temperature and loading regimes. International Virtual Journal Machines, Technologies, Materials, 9: 33-35. Available online at: http://mech-ing.com/journal/Archive/2012/9/54_Petrovic. pdf

Rager A (2003). Analysis of high rate fracture tests of polymers. Ph.D. Dissertation, Imperial College London, London, UK.

Williams JG and Hodgkinson J (1981). Crack-blunting mechanisms in impact tests on polymers. Royal Society of London A: Mathematical, Physical and Engineering Sciences, 375(1761): 231-247. 